\title{
STABILITY RESULTS FOR SOME PERIODIC FEEDBACK CONTROLLERS
}

\author{
Ömer Morgül \\ Bilkent University, Dept. of Electrical and Electronics \\ Engineering, 06800, Bilkent, Ankara, Turkey
}

\begin{abstract}
We propose two periodic feedback schemes for the stabilization of periodic orbits for one dimensional discrete time chaotic systems. These schemes can be generalized to higher dimensional systems in a straightforward way. We show that the proposed schemes achieve stabilization of a wide range of periodic orbits. The proposed schemes are quite simple and we show that any hyperbolic periodic orbit can be stabilized with these schemes. We also present some simulation results.
\end{abstract}

\section{Copyright 2005 IFAC}

Keywords: Chaos control, delayed feedback system, Pyragas controller, stability.

\section{INTRODUCTION}

The study of chaotic behaviour in dynamical systems has received great attention in recent years. The interest in using feedback control in chaotic systems mainly accelerated after the seminal work of (Ott, Grebogy and Yorke,1990), where the term "controlling chaos" was introduced. The scheme they proposed is called as OGY scheme since then. Such systems usually have many unstable periodic orbits embedded in their chaotic attractors, and as shown in (Ott, Grebogy and Yorke, 1990), some of these orbits may be stabilized by using small control input. Following this work, various chaos control techniques have been proposed, see e.g. (Chen and Dong, 1999). Among these, the delayed feedback control (DFC) scheme frst proposed in (Pyragas, 1992) and is also known as Pyragas scheme, has gained considerable attention due to its various attractive features. In this technique the required control input is basically the difference between the current and one period delayed states, multiplied by a gain. Hence if the system is already in the periodic orbit, this term vanishes. Also if the trajectories asymptotically approach to the periodic orbit, this term becomes smaller.
DFC has been successfully applied to many systems, including the stabilization of coherent modes of laser (Bielawski et. al., 1994), (Loiko, 1997); cardiac systems, (Brandt, 1997); controlling friction, (Elmer, 1998); chaotic electronic oscillators, (Pyragas, 1993). Despite its simplicity, a detailed stability analysis of DFC is very diffcult, (Pyragas, 2001), (Ushio, 1996). For some recent stability results related to DFC, see (Ushio, 1996), (Nakajima, 1997), (Pyragas, 2001), (Morgül, 2003). For more details as well as various applications of DFC, see (Pyragas, 2001), (Fradkov and Evans, 2002) and the references therein.

The DFC scheme has some inherent limitations, i.e. it cannot be applied for the stabilization of some periodic orbits, see e.g. (Ushio, 1996). To overcome the limitations of DFC, several modifcations have been proposed, see e.g. (Baba, 2002),(Socolar, 1994), (Pyragas, 2001) and the references therein. Among these, the periodic feedback law given in (Schuster and Stemmler, 1997) seems to be promising due to its simplicity. This method is known to eliminate the limitations of DFC for period 1 case, and various extensions to higher order periods are possible. In this letter we provide two such extensions. We will show 
that the proposed extensions yield stabilization of the corresponding periodic orbits.

This paper is organized as follows. First we will present some notations which will be used in the sequel. Then we will propose two periodic feedback schemes and present the related stability analysis. These results show that a wide range of periodic orbits can be stabilized with these schemes. More precisely, we will show that any hyperbolic periodic orbit can be stabilized with the proposed scheme, and this result can be generalized to higher dimensional systems in a straightforward way. Following some simulation results, Enally, we will give some concluding remarks.

\section{PROBLEM STATEMENT}

Let us consider the following one dimensional discretetime system

$$
x(k+1)=f(x(k))
$$

where $k=1,2 \ldots$ is the discrete time index, $f: \mathbf{R} \rightarrow \mathbf{R}$ is an appropriate function, which is assumed to be differentiable wherever required. We assume that the system given by (1) possesses a $T$ periodic orbit characterized by the set $\Sigma_{T}=\left\{x_{1}^{*}, x_{2}^{*}, \ldots, x_{T}^{*}\right\}$, i.e. for $x(1)=$ $x_{1}^{*}$, the iterates of (1) yields $x(2)=x_{2}^{*}, \ldots, x(T)=x_{T}^{*}$, $x(k)=x(k-T)$ for $k>T$. Let us call this orbit as an uncontrolled periodic orbit (UCPO) for future reference.

Let $x(\cdot)$ be a solution of (1). To characterize the convergence of $x(\cdot)$ to $\Sigma_{T}$, we need a distance measure, which is defned as follows. For $x_{i}^{*}$, we will use circular notation, i.e. $x_{i}^{*}=x_{j}^{*}$ for $i=j(\bmod T)$. Let us defne the following indices $(j=1, \ldots, T)$ :

$$
d_{k}(j)=\sqrt{\sum_{i=0}^{T-1}\left(x(k+i)-x_{i+j}^{*}\right)^{2}}
$$

We then defne the following distance measure

$$
d\left(x(k), \Sigma_{T}\right)=\min \left\{d_{k}(1), \ldots, d_{k}(T)\right\}
$$

Clearly, if $x(1) \in \Sigma_{T}$, then $d\left(x(k), \Sigma_{T}\right)=0, \forall k$. Conversely if $d\left(x(k), \Sigma_{T}\right)=0$ for some $k_{0}$, then it remains 0 and $x(k) \in \Sigma_{T}$, for $k \geq k_{0}$. We will use $d\left(x(k), \Sigma_{T}\right)$ as a measure of convergence to the periodic solution given by $\Sigma_{T}$.

Let $x(\cdot)$ be a solution of (1) starting with $x(1)=x_{1}$. We say that $\Sigma_{T}$ is (locally) asymptotically stable if there exists an $\varepsilon>0$ such that for any $x(1) \in \mathbf{R}$ for which $d\left(x(1), \Sigma_{T}\right)<\varepsilon$ holds, we have $\lim _{k \rightarrow \infty} d\left(x(k), \Sigma_{T}\right)=$ 0 . Moreover if this decay is exponential, i.e. the following holds for some $M \geq 1$ and $0<\rho<1,(k>1)$

$$
d\left(x(k), \Sigma_{T}\right) \leq M \rho^{k} d\left(x(1), \Sigma_{T}\right)
$$

then we say that $\Sigma_{T}$ is (locally) exponentially stable.
To stabilize the periodic orbits of (1), let us apply the following control law :

$$
x(k+1)=f(x(k))+u(k)
$$

where $u(\cdot)$ is the control input. In classical DFC, the following feedback law is used $(k>T)$ :

$$
u(k)=K(\chi(k)-\chi(k-T))
$$

where $K$ is a constant gain to be determined. It is known that the scheme given above has certain inherent limitations, see e.g. (Ushio, 1996). For example, let $\Sigma_{1}=\left\{x_{1}^{*}\right\}$ be a period 1 UCPO of (1) and set $a_{1}=f^{\prime}\left(x_{1}^{*}\right)$, where a prime denotes the derivative. It can be shown that $\Sigma_{1}$ can be stabilized with this scheme if $-3<a<1$ and cannot be stabilized if $a>1$, see (Ushio, 1996). For $\Sigma_{T}$, let us set $a_{i}=f^{\prime}\left(x_{i}^{*}\right)$. It can be shown that $\Sigma_{1}$ cannot be stabilized with this scheme if $\prod_{i=1}^{T} a_{i}>1$, see e.g. (Ushio, 1996) , (Morgül, 2003). A set of necessary and suffcient conditions to guarantee exponential stabilization can be found in (Morgül, 2003).

To overcome the limitations of DFC scheme, various modifcations have been proposed, see e.g. (Pyragas, 2001), (Fradkov and Evans, 2002). One of these schemes is the so-called periodic, or oscillating feedback, see (Schuster and Stemmler, 1997). For period 1 case, the corresponding feedback law is given by :

$$
u(k)=\varepsilon(k)(x(k)-x(k-1))
$$

where $\varepsilon(k)$ is given as :

$$
\varepsilon(k)=\left\{\begin{array}{rl}
K & k(\bmod 2)=0 \\
0 & k(\bmod 2) \neq 0
\end{array}\right.
$$

where $K$ is a constant gain to be determined. Let $\Sigma_{1}=\left\{x_{1}^{*}\right\}$ be the period 1 orbit of (1), and defne the error as $e(k)=x(k)-x_{1}^{*}$. By using the frst two iterations of (5), (7), (8) and $x_{1}^{*}=f\left(x_{1}^{*}\right)$, after linearization and considering only the frst order terms, we obtain $e(2)=a_{1} e(1), e(3)=\left(a_{1}+K\right) e(2)-K e(1)=\left(a_{1}^{2}+\right.$ $\left.\left(a_{1}-1\right) K\right) e(1)$ where $a_{1}=f^{\prime}\left(x_{1}^{*}\right)$. Clearly, if $\mid a_{1}^{2}+$ $\left(a_{1}-1\right) K \mid<1$, then $\Sigma_{1}$ is (locally exponentially) stabilizable. If $a_{1} \neq 1$, then by using the above inequality one can easily fnd a range of $K$ for which the (locally exponential) stabilization is possible. This simple analysis shows that for the case $T=1$, the inherent limitation of DFC (i.e. $-3<a_{1}<1$ ) can be avoided by using the periodic feedback law given above.

The idea given above can be generalized to the case $T=m>1$. One particular generalization is given in (Schuster and Stemmler, 1997). However, as noted in (Pyragas, 2001), the stability analysis is not clear. In the sequel, we will provide different generalizations along with their simple stability analysis. 


\section{PERIODIC CONTROLLERS}

Let us consider the case $T=m>1$, and the period $m$ orbit $\Sigma_{m}$ of (1). We will use the notation given above. Let us defne the m-iterate map $F$ as $F=f^{m}$. Clearly period $\mathrm{m}$ orbits of $f$ are equivalent to period 1 orbits of $F$, i.e. $F\left(x_{i}^{*}\right)=x_{i}^{*}, i=1,2, \ldots, m$. Now consider the following system :

$$
z(j+1)=F(z(j))+\varepsilon(j)(z(j)-z(j-1))
$$

where $\varepsilon(\cdot)$ is given by (8). Clearly, the stabilization property stated above holds if $\left|a^{2}+(a-1) K\right|<1$, where $a=F^{\prime}\left(x_{i}^{*}\right)$ for any $i=1,2, \ldots, m$. Note that we have $a=\prod_{i=1}^{m} a_{i}$.

To transform (5) into (9), let us choose $u(k)$ as follows

$$
u(k)=\varepsilon(k)(x(k-m+1)-\chi(k-2 m+1))
$$

where $\varepsilon(k)$ is given as :

$$
\varepsilon(k)=\left\{\begin{array}{l}
K k(\bmod 2 m)=0 \\
0 k(\bmod 2 m) \neq 0
\end{array}\right.
$$

Clearly, for $m=1$, both (10) and (11) reduces to (7), and (8), respectively. For the sake of clarity, we will call the scheme given by (10) and (11) as double period delayed feedback scheme (DPDFC). To see the equivalence between (9) and (5), (10)-(11), let us set $z(j)=x((j-1) m+1), j=1,2, \ldots$ If $j$ is odd by using (10)-(11) in (5), we obtain :

$$
x(j m+1)=f(x(j m))=f^{m}((j-1) m+1)
$$

which is the same as $z(j+1)=F(z(j))$ is $j$ is odd. On the other hand, if $j$ is even, similarly we obtain :

$$
\begin{aligned}
\chi(j m+1) & =f^{m}(x((j-1) m+1)) \\
& +K(x((j-1) m+1) \\
& -\chi((j-2) m+1))
\end{aligned}
$$

which is the same as $z(j+1)=F(z(j))+K(z(j)-$ $z(j-1))$ if $j$ is even. From these, it easily follows that (5), (10)-(11) is equivalent to (9). We can summarize the stability result given above as follows :

Theorem 1 : Let a period $m$ orbit of (1) be given as $\Sigma_{m}=\left\{x_{1}^{*}, \ldots, x_{m}^{*}\right\}$ and set $a_{i}=f^{\prime}\left(x_{i}^{*}\right), i=1,2, \ldots, m$, $a=\prod_{i=1}^{m} a_{i}$. The DPDFC scheme given by (5), (10)(11) is (locally exponentially) stable if and only if

$$
\left|a^{2}+(a-1) K\right|<1
$$

Proof : Note that the local exponential stability is equivalent to the stability of the linearized system. The proof of the theorem then easily follows from the results stated above.

Remark 1 : Note that although the stability condition given by (14) is similar to the one given in(Schuster and Stemmler, 1997), the form of both (10) and (11) are different than the ones given in (Schuster and Stemmler, 1997).

Remark 2 : Note that when $\left|a^{2}+(a-1) K\right|>1, \Sigma_{m}$ is not stable, and when $\left|a^{2}+(a-1) K\right|=1$, the analysis given above is not conclusive.

Remark 3 : If $a \neq 1$, then one can always fnd a range of $K$ such that (14) holds. Since this condition is expected to hold for most of the chaotic orbits, the proposed methodology is applicable to the stabilization of periodic orbits in a large class of chaotic systems. $\square$.

In the sequel, we will provide another possible generalization of (7), (8) to the case $T=m>1$. In this scheme, the control input is given as :

$$
u(k)=\varepsilon(k)(x(k)-\chi(k-m))
$$

where $\varepsilon$ is given as :

$$
\varepsilon(k)=\left\{\begin{array}{r}
K k(\bmod (m+1))=0 \\
0 k(\bmod (m+1)) \neq 0
\end{array}\right.
$$

Clearly, for $m=1$, both (15) and (16) reduces to (7), and (8), respectively. For the sake of clarity, we will call the scheme given by (15) and (16) as single period delayed feedback scheme (SPDFC). However, unlike the previous case, this scheme is not equivalent to (9), hence the stability analysis given above is not valid. For the stability of this scheme, let $\Sigma_{m}$ be the period $m$ orbit of (1), and set $a_{i}=f^{\prime}\left(x_{i}^{*}\right), i=1,2, \ldots, m, a=$ $\prod_{i=1}^{m} a_{i}$. In the sequel, we will use the circular notation for $x_{i}^{*}$ and $a_{i}$, i.e. $x_{i}^{*}=x_{j}^{*}, a_{i}=a_{j}$ if $i=j(\bmod m)$. By using $x_{j+1}^{*}=f\left(x_{j}^{*}\right)$ in (5), (15), (16), by using linearization and considering only the frst order terms we obtain $(i=0,1, \ldots, j=1,2, \ldots)$ :

$$
\begin{gathered}
x(j+1+i(m+1))-x_{j+i+1}^{*}= \\
a_{i+j}\left(x(j+i(m+1))-x_{i+j}^{*}\right) \\
x((i+1)(m+1)+1)-x_{i+2}^{*}= \\
\quad\left(a\left(a_{i+1}+K\right)\right. \\
\quad-K)\left(x(i(m+1)+1)-x_{i+1}^{*}\right)
\end{gathered}
$$

For the sake of simplicity, let us defne the following $(j=1,2, \ldots, m)$ :

$$
\begin{gathered}
p_{j}=a\left(a_{j}+K\right)-K \quad, \quad p=\prod_{i=1}^{m} p_{j} \\
e(i)=x((i-1)(m+1)+1)-x_{i}^{*}
\end{gathered}
$$

By using (18)-(20), we easily obtain the following $(i=1,2, \ldots, j=0,1, \ldots)$ :

$$
e(i+j m)=p^{j} e(i)
$$

Clearly we have stability of $\Sigma_{m}$ if $|p|<1$. We summarize this result as follows.

Theorem 2 : Let a period $m$ orbit of (1) be given as $\Sigma_{m}=\left\{x_{1}^{*}, \ldots, x_{m}^{*}\right\}$ and set $a_{i}=f^{\prime}\left(x_{i}^{*}\right), i=1,2, \ldots, m$, 
$a=\prod_{i=1}^{m} a_{i}$. The SPDFC scheme given by (5), (15)(16) is (locally exponentially) stable if and only if $|p|<1$ where $p$ is given by (19).

Proof : The proof easily follows from the analysis given above.

Remark 4 : Note that when $|p|>1, \Sigma_{m}$ is unstable and when $|p|=1$ the analysis given above is not conclusive. $\square$

Remark 5 : If $a \neq 1$, then note that for $K_{j}=a a_{j} /(1-$ $a$ ), we have $p_{j}=0$, hence $p=0$. Therefore by continuity arguments it follows that there exists constants $K_{j}^{-}<K_{j}<K_{j}^{+}$such that for $K_{j}^{-}<K<K_{j}^{+}$we have $|p|<1$. Hence, for $a \neq 1$, one can always fnd a set of intervals for the gain $K$ such that SPDFC scheme proposed above stabilizes $\Sigma_{m}$.

Remark 6 : We note that, although we presented our schemes for one dimensional systems, they can easily be generalized to higher dimensional systems easily in a straightforward way. In the next remark, we will keep this point in mind. $\square$

Remark 7 : To see the improvement we obtained by using the proposed schemes over the classical DFC for the stabilization of $\Sigma_{m}$, let us consider the latter. It is known that classical DFC scheme has some inherent limitations, and it can be shown that it cannot stabilize $\Sigma_{m}$ if the number of real eigenvalues of $J_{i}$ (which is the multiplication of the Jacobians of $f$ evaluated at the periodic points) greater than 1 is odd, see e.g. (Ushio, 1999), (Morgül, 2003). Note that this condition is not satisfed in many chaotic orbits, and classical DFC cannot be used in their stabilization. Also note that even if this necessary condition is satisfed, stabilization by classical DFC is not guaranteed, see (Morgül, 2003). On the other hand, the schemes presented in this paper always yield stabilization provided that $\lambda=1$ is not an eigenvalue of $J_{i}$, (i.e. for one dimensional case, $J_{i}=a \neq 1$ ). Since we are mainly concerned with the stabilization of unstable periodic orbits, this condition most likely holds in most of the periodic orbits, hence we can safely state that practically all periodic orbits can be stabilized with this approach. We also note that having an eigenvalue at $\lambda=1$ maybe considered as a nongeneric case, hence from this point of view we may also argue that almost all of the unstable periodic orbits can be stabilized by the proposed schemes. Note that this property is related to the hyperbolic behaviour of the periodic orbits. Recall that a periodic orbit $\Sigma_{m}$ is called hyperbolic if none of the eigenvalues of $J_{i}$ are on the unit disc, see e.g. (Devaney, 1987). Hence, the results (i.e. Theorems 1 and 2) imply that any hyperbolic periodic orbit can be stabilized by the proposed schemes. $\square$

\section{SIMULATION RESULTS}

For the simulation results, we frst consider the logistic map given by $f(x)=4 x(1-x)$. It is well-known that this map has chaotic solutions and periodic orbits of all orders. Two true period 3 orbits of this map can be computed as $\Sigma_{3-}=\{0.413175,0.969846,0.116977\}$, $\Sigma_{3+}=\{0.611260,0.950484,0.188255\}$. For $\Sigma_{3-}$, we have $a=-8$, and by using (14), it follows that exponential stability holds for $7<K<7.22$. Since the stabilization is only local, the DPDFC will work when the actual orbit of (1) is suffciently close to the periodic solution. Hence, we apply the control law given by (10)-(11) when $d\left(x(k), \Sigma_{3-}\right)<\varepsilon$, otherwise we set $u(k)=0$, where $\varepsilon>0$ is a measure of domain of attraction. To evaluate the exact domain of attraction is very diffcult, but by extensive numerical simulations we End that for $K=7.11$, we have $\varepsilon=0.04$. Since the solutions of (1) are chaotic, eventually the control law given above will be effective and the stabilization will be achieved for any $x(1) \in(0,1)$. Our simulations show exponential stabilization for any $x(1) \in(01)$, and a typical result for $x(1)=0.3$ is shown in Figures 1 and 2 . Note that $d\left(x(k), \Sigma_{3-}\right)$ versus $k$ and $u(k)$ versus $k$ graphs are plotted in Figures 1 and 2, respectively. For $\Sigma_{3+}$, we have $a=8$, and since $a>1$, this orbit cannot be stabilized by DFC, (Ushio, 1996), (Morgul, 2003). By using (14), it follows that exponential stability holds for $-9.28<K<-9$. For this case, we choose $K=-9.14$, and by extensive numerical simulations we fnd that we have $\varepsilon=0.05$. The result of a particular simulation with $x(1)=0.2$ is shown in Figures 3 and 4 , where we plotted $d\left(x(k), \Sigma_{3+}\right)$ versus $k$ and $u(k)$ versus $k$ graphs in Figures 3 and 4 , respectively . As can be seen, exponential stabilization occurs.

To test the SPDFC scheme, we used the tent map given by $f(x)=1.9 x$ for $x<0.5$, and $f(x)=1.9(1-$ $x$ ) for $x>0.5$. It is well-known that this map has chaotic solutions and periodic orbits of all orders. Two true period 3 orbits of this map can be computed as $\Sigma_{3_{-}}=\{0.872757,0.241761,0.459345\}, \quad \Sigma_{3+}=$ $\{0.846390,0.291858,0.554531\}$. For $\Sigma_{3-}$, we have $a_{1}=-1.9, a_{2}=a_{3}=1.9, a=-6.859$, and by using Theorem 2, it follows that exponential stability holds for $-1.683<K<-1.633$, or $1.6582<K<1.6584$. For this case, we choose $K=-1.65$, and by extensive numerical simulations we fnd that we have $\varepsilon=0.1$. Our simulations show exponential stabilization for any $x(1) \in\left(\begin{array}{ll}0 & 1\end{array}\right)$, and a typical result for $x(1)=0.2$ is shown in Figures 5 and 6 . Note that $d\left(x(k), \Sigma_{3-}\right)$ versus $k$ and $u(k)$ versus $k$ graphs are plotted in Figures 5 and 6, respectively. For $\Sigma_{3+}$, we have $a_{1}=a_{3}=-1.9$, $a_{2}=1.9, a=6.859$, and since $a>1$, this orbit cannot be stabilized by DFC, (Ushio, 1996), (Morgul, 2003)

. By using Theorem 2, it follows that exponential stability holds for $2.19<K<2.25$, or $-2.2245<$ $K<-2.2240$. For this case, we choose $K=2.2$, and by extensive numerical simulations we fnd that we 
have $\varepsilon=0.1$. The result of a particular simulation with $x(1)=0.2$ is shown in Figures 7 and 8, where we plotted $d\left(x(k), \Sigma_{3+}\right)$ versus $k$ and $u(k)$ versus $k$ graphs in Figures 7 and 8, respectively. As can be seen, exponential stabilization occurs.

\section{CONCLUSION}

In conclusion, we proposed two generalizations of periodic delayed feedback law given by (7) to arbitrary period case for one dimensional discrete time chaotic systems. We showed that under a mild condition $(a \neq$ 1) local exponential stabilization of any periodic orbit is possible. These schemes can be generalized to higher dimensional systems in a straightforward way. We showed that the proposed schemes achieve stabilization of a wide range of periodic orbits. The proposed schemes are quite simple and we showed that any hyperbolic periodic orbit can be stabilized with these schemes. This shows that the prosed schemes eliminate the basic limitations of classical DFC and can be applied to a broad class of chaotic systems. We also presented some simulation results.

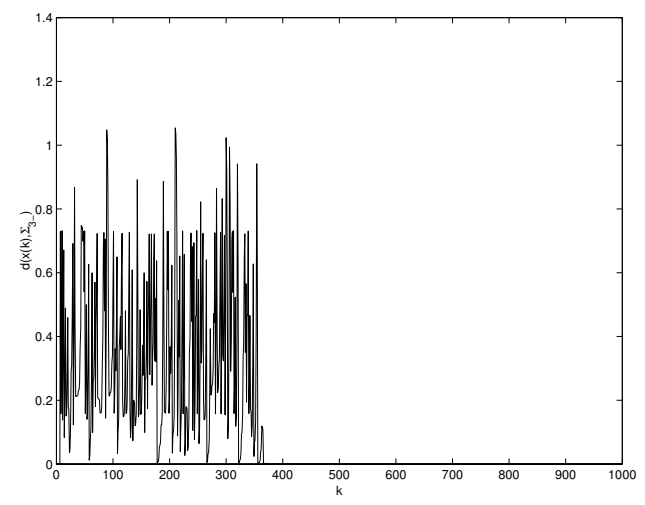

Fig. 1. DPDFC scheme, $d\left(x(k), \Sigma_{3-}\right)$ vs. $k$ for logistic map

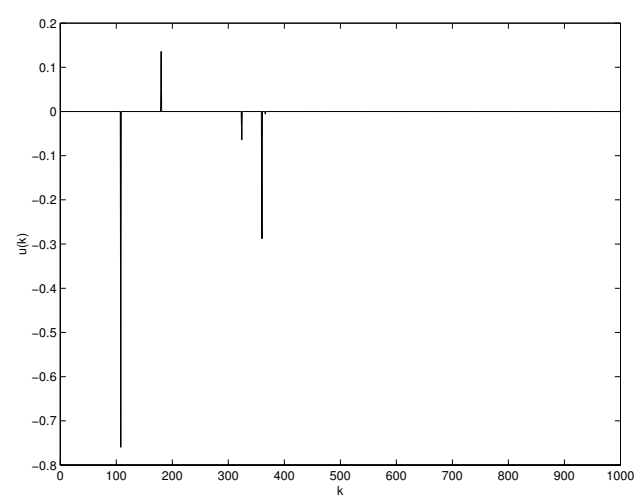

Fig. 2. DPDFC scheme, $u(k)$ vs. $k$ for the stabilization of $\Sigma_{3-}$ for logistic map,

\section{REFERENCES}

Baba, N, A. Amann, and E. Schöll (2002), Giant improvement of time delayed feedback control by

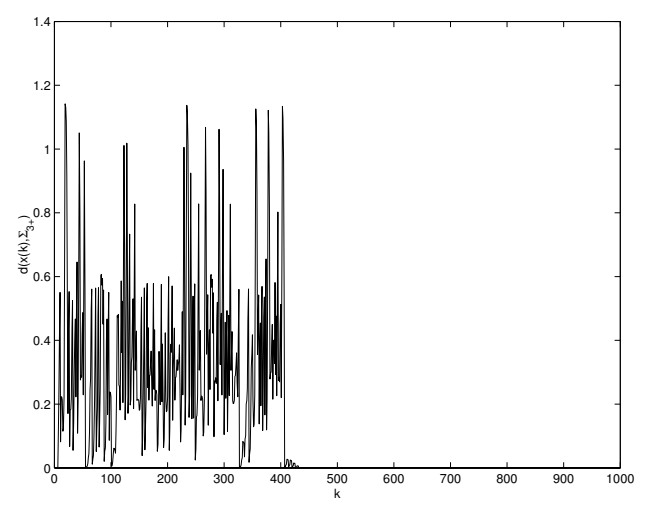

Fig. 3. DPDFC scheme, $d\left(x(k), \Sigma_{3+}\right)$ vs. $k$ for logistic map

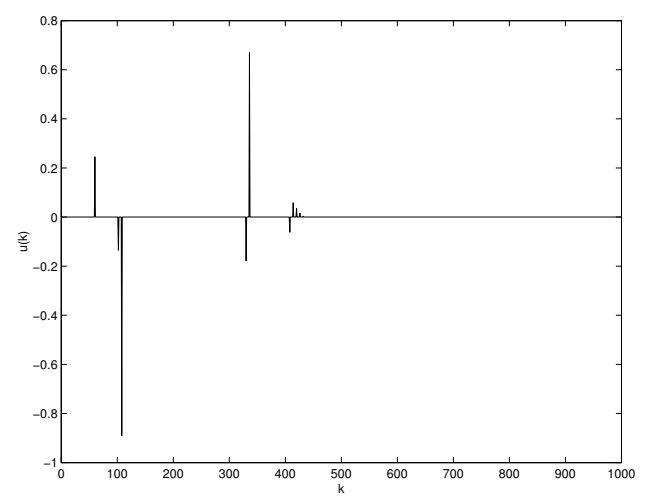

Fig. 4. DPDFC scheme, $u(k)$ vs. $k$ for the stabilization of $\Sigma_{3+}$ for logistic map,

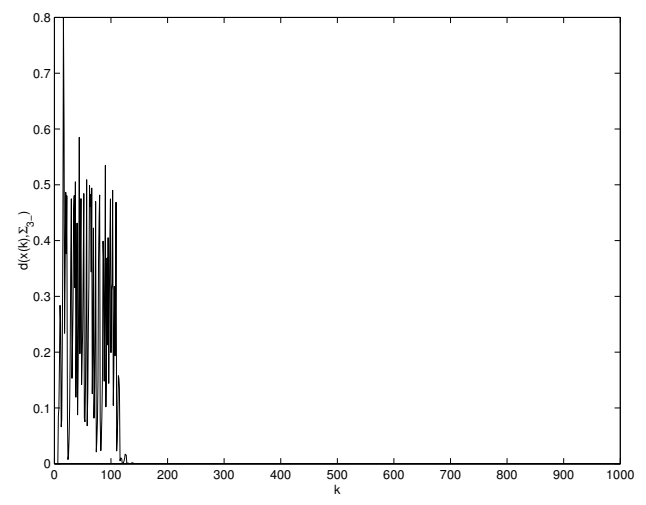

Fig. 5. SPDFC scheme, $d\left(x(k), \Sigma_{3-}\right)$ vs. $k$ for tent map spatio-temporal fltering, Phys. Rev. Lett., 89 41014104.

Bielawski, S., D. Derozier, and P. Glorieux (1993), Experimental characterization of unstable periodic orbits by controlling chaos, Phys. Rev. A, 47 R2492R2495,

Brandt, M. E., H. T. Shih, and G. Chen (1997), Linear time delay feedback control of a pathological rhythm in a cardiac conduction model, Phys. Rev. E, 56 R1334-R1337.

Chen, G., and X. Dong, From Chaos to Order 


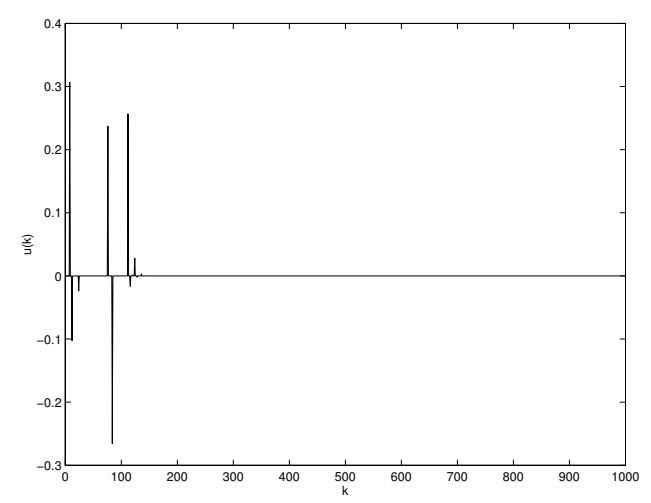

Fig. 6. SPDFC scheme, $u(k)$ vs. $k$ for the stabilization of $\Sigma_{3-}$ for tent map,

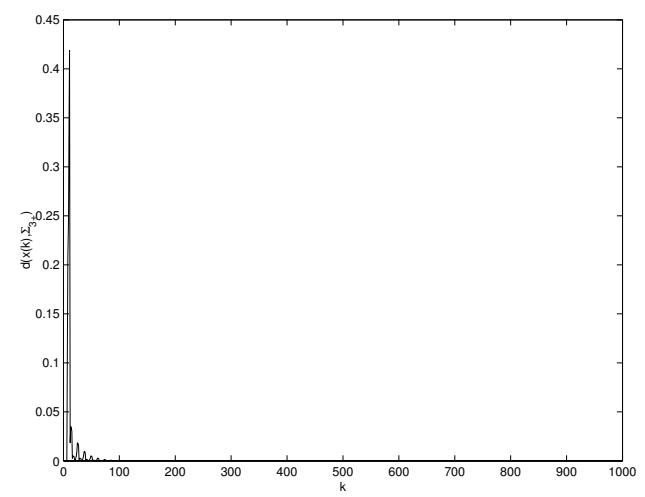

Fig. 7. SPDFC scheme, $d\left(x(k), \Sigma_{3+}\right)$ vs. $k$ for tent map

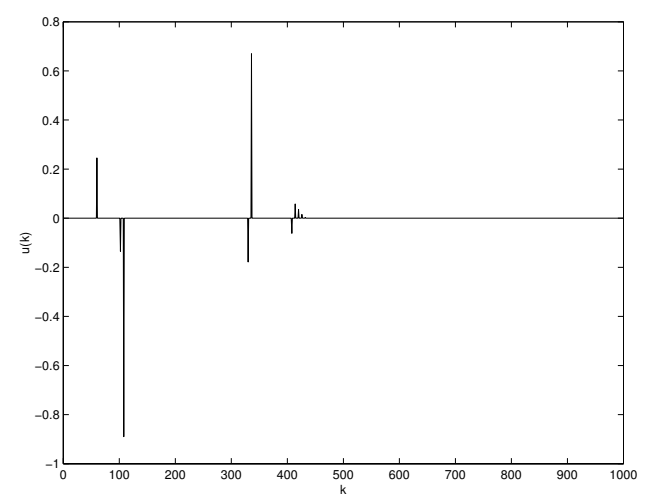

Fig. 8. SPDFC scheme, $u(k)$ vs. $k$ for the stabilization of $\Sigma_{3+}$ for tent map,

: Methodologies, Perspectives and Applications (1999), World Scientifc, Singapore.

Devaney, R. L. (1987(, Chaotic Dynamical Systems, Addison-Wesley, Redwood City.

Elmer, F. J. (1998) Controlling friction, Phys. Rev. E, 57 R4903-R4906.

Fradkov, A. L., and R. J. Evans (2002) Control of Chaos : Survey 1997-2000," Proceedings of 15th IFAC World Congress, 21-26 July 2002, Barcelona, Spain, pp. 143-154.
Just, W., T. Bernard, M. Ostheimer, E. Reibold, and H. Benner (1997), Mechanism of time delayed feedback control, Phys. Rev. Lett., 78, 203-206.

Loiko, N. A., A. V. Naumenko, and S. I. Turovets (1997), Effect of Pyragas feedback on the dynamics of a Q-switched laser, J. Exper. Theor. Physics, 85 pp. 827-834.

Morgül, Ö. (2003) On the stability of delayed feedback controllers, Phys. Lett. A, 314, pp. 278-285.

Nakajima, H. (1997) On analytical properties of delayed feedback control of chaos, Phys. Lett. A, 232, 207-210.

Ott, E., C. Grebogi, and J. A. Yorke (1990) Controlling Chaos, Phys. Rev. Lett., 64, pp. 1196-1199.

Pyragas, K. (1992) Continuous control of chaos by self-controlling feedback Phys. Lett. A., 170, pp. 421-428.

Pyragas, K. (2001) Control of chaos via an unstable delayed feedback controller, Phys. Rev. Lett., 86 pp. 2265-2268.

Pyragas, K., and A. Tamašiavięius (1993), Experimental control of chaos by delayed self controlling feedback, Phys. Lett. A, 180 99-102. (1999).

Schuster, H. G., and M. B. Stemmler (1997) Control of chaos by oscillating feedback, Phys. Rev. E, 56, pp. 6410-6417.

Socolar, J. E. S., D. W. Sukow, and D. J. Gauthier (1994) Stabilizing unstable periodic orbits in fast dynamical systems, Phys. Rev. E, 50 3245-3248.

Ushio, T. (1996) Limitation of delayed feedback control in nonlinear discrete-time systems, IEEE Trans. Circuits Syst. I, Fundam. Theory Appl., 43 pp. 815-816. 